\title{
CALIPSO polar stratospheric cloud observations: second-generation detection algorithm and composition discrimination
}

\author{
M. C. Pitts ${ }^{1}$, L. R. Poole ${ }^{2}$, and L. W. Thomason ${ }^{1}$ \\ ${ }^{1}$ NASA Langley Research Center, Hampton, Virginia, USA \\ ${ }^{2}$ Science Systems and Applications, Incorporated, Hampton, Virginia, USA
}

Received: 9 January 2009 - Published in Atmos. Chem. Phys. Discuss.: 27 March 2009

Revised: 13 August 2009 - Accepted: 21 September 2009 - Published: 12 October 2009

\begin{abstract}
This paper focuses on polar stratospheric cloud (PSC) measurements by the CALIOP (Cloud-Aerosol LIdar with Orthogonal Polarization) lidar system onboard the CALIPSO (Cloud-Aerosol Lidar and Infrared Pathfinder Satellite Observations) spacecraft, which has been operating since June 2006. We describe a second-generation PSC detection algorithm that utilizes both the CALIOP 532-nm scattering ratio (ratio of total-to-molecular backscatter coefficients) and 532-nm perpendicular backscatter coefficient measurements for cloud detection. The inclusion of the perpendicular backscatter measurements enhances the detection of tenuous PSC mixtures containing low number densities of solid (likely nitric acid trihydrate, NAT) particles and leads to about a $15 \%$ increase in PSC areal coverage compared with our original algorithm. Although these low number density NAT mixtures would have a minimal impact on chlorine activation due to their relatively small particle surface area, these particles may play a significant role in denitrification and therefore are an important component of our PSC detection. In addition, the new algorithm allows discrimination of PSCs by composition in terms of their ensemble backscatter and depolarization in a manner analogous to that used in previous ground-based and airborne lidar PSC studies. Based on theoretical optical calculations, we define four CALIPSO-based composition classes which we call supercooled ternary solution (STS), ice, and Mix1 and Mix2, denoting mixtures of STS with NAT particles in lower or higher number densities/volumes, respectively. We exam-
\end{abstract}

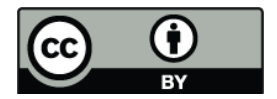

Correspondence to: M. C. Pitts (michael.c.pitts@nasa.gov) ine the evolution of PSCs for three Antarctic and two Arctic seasons and illustrate the unique attributes of the CALIPSO PSC database. These analyses show substantial interannual variability in PSC areal coverage and also the well-known contrast between the Antarctic and Arctic. The CALIPSO data also reveal seasonal and altitudinal variations in Antarctic PSC composition, which are related to changes in $\mathrm{HNO}_{3}$ and $\mathrm{H}_{2} \mathrm{O}$ observed by the Microwave Limb Sounder on the Aura satellite.

\section{Introduction}

In the two plus decades since it became evident that polar stratospheric clouds (PSCs) play a major role in the springtime depletion of polar ozone, the detailed composition and morphology of PSCs has been an area of interest. A historical overview and a comprehensive discussion of PSC microphysics and chemistry can be found in the recent paper by Lowe and MacKenzie (2008). Although our PSC knowledge base is substantial, there is still considerable uncertainty in specific key areas related to their composition and formation. Probably the least well understood of these is the nucleation process responsible for the formation of nitric acid trihydrate (NAT) particles and the circumstances under which NAT formation and growth leads to stratospheric denitrification. This limits our ability to accurately represent PSC formation in chemical transport models that are used to predict the future evolution of polar ozone abundance and to detect the early signs of recovery. This is of particular concern for projections of future ozone loss in the Arctic where winter vortex temperatures generally hover near the threshold for PSC

Published by Copernicus Publications on behalf of the European Geosciences Union. 
formation and even a modest drop in stratospheric temperatures could lead to more significant ozone depletion.

New insight into PSC morphology is being provided by the on-going Cloud-Aerosol Lidar and Infrared Pathfinder Satellite Observations (CALIPSO) spaceborne mission (Winker et al., 2007). In addition, CALIPSO has the potential to provide considerable insight into the key remaining questions regarding PSC composition and formation mechanisms. The lidar on CALIPSO, CALIOP (Cloud-Aerosol LIdar with Orthogonal Polarization), is an excellent instrument for observing PSCs (Pitts et al., 2007) and has already produced an extensive set of PSC observations covering three Antarctic and two Arctic "seasons." These observations provide unprecedented spatial and temporal resolution that greatly reduces sampling bias characteristic of occultation instruments that were the basis of past space-based studies of PSC characteristics (Poole and Pitts, 1994; Fromm et al., 2003). The dramatic scale of these differences was demonstrated by Pitts et al. (2007). The Pitts et al. (2007) analyses were based on a preliminary CALIPSO PSC detection algorithm that was based on a threshold approach using only scattering ratio (total backscatter/molecular backscatter) measurements at $532 \mathrm{~nm}$.

It was clear even during the development of the original detection algorithm (hereafter referred to as v1) that some PSCs that were below the scattering ratio threshold (hence, not identified as PSCs by v1) were outliers in the CALIOP perpendicular backscatter data. These clouds most likely include very low number densities of non-spherical particles and show only very small enhancements in scattering ratio relative to the background aerosol. On the other hand, the 532-nm perpendicular backscatter coefficient measurement is very sensitive to the presence of non-spherical solid particles (NAT and ice), but not to spherical liquid particles (aerosol and supercooled ternary solution, or STS). As a result, while the magnitude of the enhancement in perpendicular backscatter is small, very optically-thin PSCs containing solid particles can frequently be identified. To fully exploit the CALIOP PSC data for studies of NAT formation processes, we must maximize our ability to detect the presence of NAT, particularly at low number densities/volumes. Therefore, adding the CALIOP perpendicular backscatter coefficient measurements to our second-generation algorithm is essential. In addition, as demonstrated below, the inclusion of the perpendicular backscatter measurement enables the discrimination of PSCs by composition.

Our approach follows a long history of using polarizationsensitive lidar data to study PSC composition, dating back to papers by Poole and McCormick (1988), Browell et al. (1990), and Toon et al. (1990), from which originated the commonly used hierarchy of PSC "types" - type 1a for NAT, type $1 \mathrm{~b}$ for STS, and type 2 for $\mathrm{H}_{2} \mathrm{O}$ ice. More recent studies (e.g., Gobbi et al., 1998; Toon et al., 2000; Biele et al., 2001; Hu et al., 2002; Adriani et al., 2004; Massoli et al., 2006) indicate that such distinct "typing" of PSCs is an oversimplification, with the clouds often occurring as mixtures of liquid and solid particles. Hence, we explore the CALIOP measurement space using a theoretical optical model for simple notional mixtures of STS and NAT or ice particles, and then use these calculations in conjunction with statistical analyses of the CALIOP aerosol depolarization data to define new CALIPSO-based PSC composition classes. We then apply the detection and composition classification algorithms to CALIOP data from the 2006-2008 Antarctic and 2006/2007-2007/2008 Arctic winter seasons to illustrate the capabilities of CALIPSO for characterizing PSCs.

\section{Second-generation detection algorithm}

In optical measurements, PSCs appear as enhancements above the background aerosol ensemble which contains both geophysical variability and noise. Under most circumstances, the separation between aerosol and PSCs is not distinct, but rather a continuum and most detection methods rely on identification of PSCs as statistically significant enhancements above a threshold derived from the background aerosol values. Simple threshold approaches based on lidar scattering ratio and depolarization ratio measurements are commonly utilized for PSC detection in ground-based and airborne lidar applications (e.g. Toon et al., 2000; Massoli et al., 2006). We adopted a similar approach for PSC detection in the spaceborne CALIOP data. Our v1 PSC detection algorithm (Pitts et al., 2007) identified PSCs as statistical outliers of the background aerosol population using only the CALIOP scattering ratio measurements at $532 \mathrm{~nm}$. Our second-generation detection algorithm extends that approach to include a number of enhancements, the most significant being the addition of the CALIOP perpendicular backscatter coefficient measurements at $532 \mathrm{~nm}$, to the detection process. Other notable enhancements include an improved successive spatial averaging scheme, a requirement for PSCs to be spatially coherent, and higher vertical resolution. In this section, we describe the second-generation algorithm, with emphasis on the new elements of the algorithm and their impact on PSC detection.

\subsection{Data preparation}

All analyses presented here are based on v2.01 CALIOP Level 1B Profile data products available through the NASA Langley Atmospheric Science Data Center (ASDC) (http: //eosweb.larc.nasa.gov/). The primary CALIOP Level 1B data products used in our analyses are the total (parallel + perpendicular) attenuated backscatter at $532 \mathrm{~nm}, \beta_{532}^{\prime}$, and the perpendicular component of the attenuated backscatter at $532 \mathrm{~nm}, \beta_{\text {perp }}^{\prime}$. Similar to Pitts et al. (2007), profiles of total volume backscatter coefficient at $532 \mathrm{~nm}, \beta_{532}$, and the perpendicular component of the volume backscatter 
coefficient at $532 \mathrm{~nm}, \beta_{\text {perp }}$, are derived from the Level 1B attenuated backscatter profiles by applying a first-order correction for molecular and ozone attenuation based on GEOS5 molecular and ozone number density profiles provided in the CALIOP Level 1B data files. A description of the operational algorithms used to generate the CALIOP attenuated backscatter profiles and all other Level 1B data products can be found in Hostetler et al. (2006). Assessments of the CALIOP calibration, performance, and data quality have been documented elsewhere in the literature (e.g., Powell et al., 2009; Hunt et al., 2009; Winker et al., 2007; McGill et al., 2007).

PSC detection is limited to nighttime CALIOP observations because higher levels of background light present during daytime significantly reduce the measurement signal-tonoise ratio (SNR) and, hence, the PSC detection sensitivity. Since the spatial resolution of the Level 1B CALIOP data products is altitude dependent (Winker et al., 2007), we first smooth the data to a uniform grid with 5-km horizontal and $180-\mathrm{m}$ vertical resolution. In $\mathrm{v} 1$, this initial horizontal smoothing was accomplished by application of a $5-\mathrm{km}$ median filter. However, due to the noise characteristics of the CALIOP data, the median statistic is smaller in magnitude than the mean and produces backscatter coefficient values that are systematically biased low. Since PSC detection is based on relative increases in scattering ratio, the low bias did not impact cloud detection and, in fact, the median filter was more effective than the mean at eliminating space environment radiation-induced noise spikes that are common in the data. Nonetheless, for applications where absolute values of the lidar optical measurements are critical, such as PSC composition discrimination, the median statistic is not appropriate. Therefore, in the second-generation algorithm we apply $5-\mathrm{km}$ arithmetic averaging for the initial horizontal smoothing of the data. As a consequence, there are more isolated 'false positive' PSCs identified than in the previous version and we have developed a spatial coherence test as a means of excluding these from consideration as PSCs. The spatial coherence test is described in more detail in Sect. 2.3.

\subsection{Cloud detection}

PSCs are identified in the CALIOP data record as statistical outliers of the background stratospheric aerosol population in terms of either scattering ratio at $532 \mathrm{~nm}, \mathrm{R}_{532}$, or $\beta_{\text {perp }}$. Here, $R_{532}$ is defined as

$\mathrm{R}_{532}=\frac{\beta_{532}}{\beta_{m}}$

where $\beta_{532}$ is the total volume backscatter and $\beta_{m}$ is the molecular backscatter coefficient at $532 \mathrm{~nm}$. $\beta_{m}$ is calculated from the GEOS-5 molecular density profiles provided in the CALIOP Level 1B data files and a theoretical value for the molecular scattering cross section as described in Hostetler et al. (2006). The $5-\mathrm{km}$ horizontal by $180-\mathrm{m}$ ver- tical resolution CALIOP data is processed through the PSC detection algorithm on an orbit-by-orbit basis for each day during a PSC "season" (May-October in the Antarctic and December-March in the Arctic). Individual profiles of $\mathrm{R}_{532}$ and $\beta_{\text {perp }}$ are scanned from the top altitude $(30 \mathrm{~km})$ downward. Data points that exceed PSC detection thresholds in either $\mathrm{R}_{532}$ or $\beta_{\text {perp }}$ are tentatively identified as cloud features. Optically-thick features such as ice clouds are easily detected at the standard 5-km by $180-\mathrm{m}$ resolution. For more tenuous clouds, additional averaging is required to obtain the SNR necessary to detect these features above the background noise levels. To identify all cloud features within a given scene at the maximum spatial resolution possible, we employ a successive horizontal averaging scheme. Four passes are made through each nighttime orbit of data, constructing profiles of $\mathrm{R}_{532}$ and $\beta_{\text {perp }}$ at increasingly coarser spatial resolutions of $5,15,45$, and $135 \mathrm{~km}$. At each spatial scale, new cloud detection thresholds are calculated and the profiles are scanned for the presence of clouds. The backscatter data from those regions identified as cloud are removed from subsequent processing. As a consequence, features found at higher spatial resolution (i.e. less averaging) are not included in the profiles of $\mathrm{R}_{532}$ and $\beta_{\text {perp }}$ at coarser resolution (i.e. more averaging). No additional vertical averaging is performed and the data retain $180-\mathrm{m}$ vertical resolution for all horizontal spatial scales. Due to the relatively high level of noise in the perpendicular backscatter channel, we do not identify PSCs using $\beta_{\text {perp }}$ at $5-\mathrm{km}$ resolution.

Cloud features identified during the detection process must also pass a spatial coherence test to minimize false positives. False positives are generally associated with noise spikes that occur as spatially isolated events while clouds typically exhibit coherent structure over spatial scales on the order of hundreds of meters in the vertical and tens of kilometers in the horizontal. The spatial coherence test requires at least 7 of the 15 points in a 5-point horizontal by 3-point vertical box centered on the candidate cloud feature to have been identified as PSCs at a finer averaging scale or a total of 11 points to either exceed the current PSC detection threshold or have been identified as cloudy at a finer averaging scale. While this test is somewhat heuristic, we feel that this combination of requirements balances the desire to eliminate obvious false positives while retaining apparent cloud elements which typically happens near the edges of extensive clouds. Analyses of the CALIOP Antarctic PSC database from early May and late October (when no PSCs observations are expected) indicate that the false positive rate is less than $0.1 \%$.

\subsection{Threshold computation}

The definition of the PSC thresholds for $\mathrm{R}_{532}$ and $\beta_{\text {perp }}$ is a critical element of the detection algorithm. This process is illustrated graphically in Fig. 1 using a single orbit of CALIOP measurements between potential temperatures of 400 and $500 \mathrm{~K}$. The background aerosol population is represented 

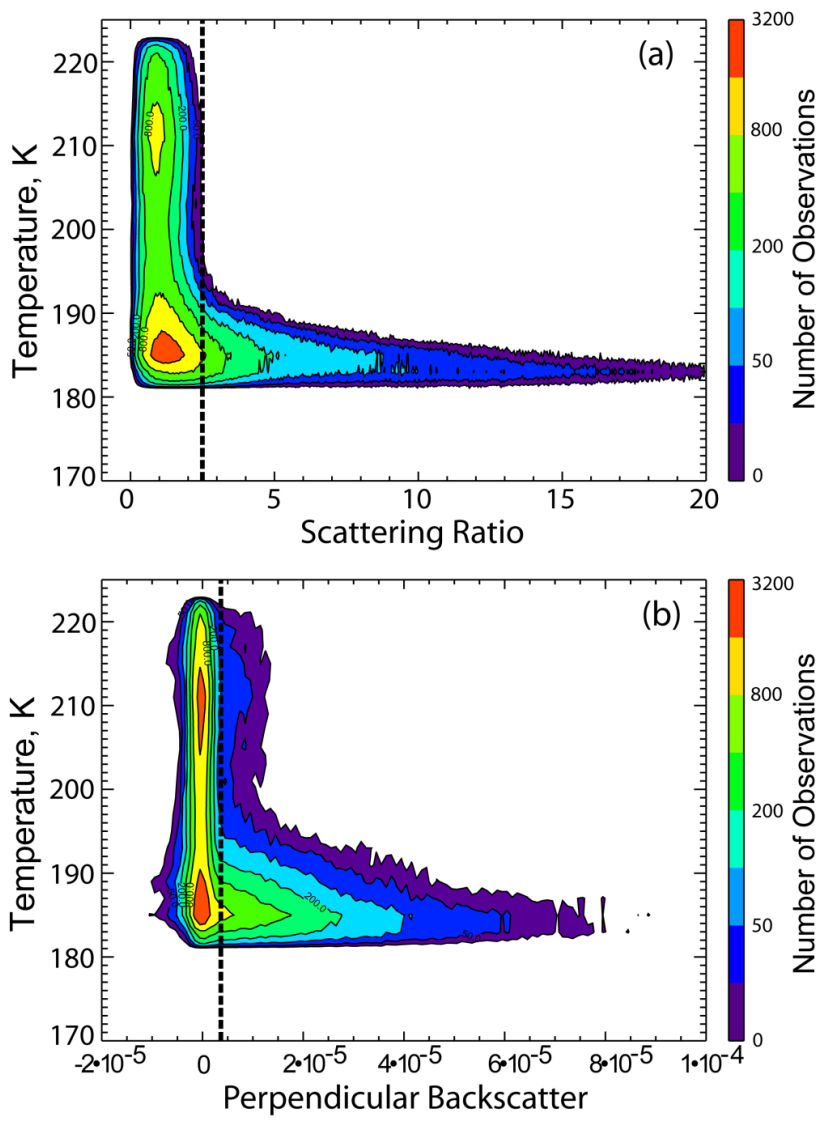

Fig. 1. Ensemble of (a) 532-nm scattering ratio and (b) 532-nm perpendicular backscatter measurements as a function of observed temperature for the 400-500 K potential temperature layer from an individual orbit of CALIPSO data on 1 August 2006. The black dashed lines indicate the scattering ratio and perpendicular backscatter coefficient thresholds for PSC detection.

by the vertical ensemble of observations centered near $\mathrm{R}_{532}=1.0$ (Fig. 1a) and $\beta_{\text {perp }}=0.0$ (Fig. 1b), while PSCs appear as the large enhancements in $\mathrm{R}_{532}$ and $\beta_{\text {perp }}$ observed at temperature colder than $\sim 195 \mathrm{~K}$. To avoid cloud contamination in computing the threshold, the background aerosol population is characterized by the ensemble of CALIOP measurements that occur at temperatures above $200 \mathrm{~K}$. Cloud detection thresholds for both $\mathrm{R}_{532}$ and $\beta_{\text {perp }}$ lie near the upper limit of the noise envelope on the background aerosol and any measurement that exceeds the threshold is clearly a statistical outlier. In the $\mathrm{v} 1$ algorithm, the $\mathrm{R}_{532}$ threshold was defined as the 99.5 percentile of the daily background aerosol ensemble. In the second-generation algorithm, the more frequent occurrence of noise spikes due to the use of mean statistics instead of medians in the Level 1B data preprocessing invalidates the 99.5 percentile as a robust $R_{532}$ threshold. The 99.5 percentile statistic also turns out to be a poor choice for the $\beta_{\text {perp }}$ threshold due to the magnitude and character of the noise in the CALIOP $\beta_{\text {perp }}$ channel. Instead, we found that a detection threshold defined as the median of the background aerosol ensemble plus four times its median deviation works well for both $\mathrm{R}_{532}$ and $\beta_{\text {perp }}$ and is stable from day to day. The detection thresholds using this definition are shown in Fig. 1.

Using the approach outlined above, $\mathrm{R}_{532}$ and $\beta_{\text {perp }}$ PSC detection thresholds are calculated for each day of CALIOP observations within the PSC seasons (May-October in the Antarctic and December-March in the Arctic). Due to the change in character of the CALIOP measurement noise with altitude (i.e. decreasing SNR with altitude), a single daily $\mathrm{R}_{532}$ or $\beta_{\text {perp }}$ threshold is not adequate for the extended altitude range $(8-30 \mathrm{~km})$ of data used in our PSC analyses. To account for this altitude dependence, daily thresholds are calculated for five overlapping $100 \mathrm{~K}$-thick potential temperature layers covering nearly the entire altitude range [400$500 \mathrm{~K}, 450-550 \mathrm{~K}, 500-600 \mathrm{~K}, 550-650 \mathrm{~K}$, and $600-700 \mathrm{~K}]$. Within each layer, the background aerosol properties are characterized and threshold values for $\mathrm{R}_{532}$ and $\beta_{\text {perp }}$ are determined. To avoid discontinuities, vertical threshold profiles are produced by linear interpolation between the 100$\mathrm{K}$ potential temperature layer values. Overall, the thresholds are well-behaved with the $\mathrm{R}_{532}$ threshold for a given potential temperature layer being nearly constant throughout a season ( $<5 \%$ variation), while the $\beta_{\text {perp }}$ thresholds exhibit a small seasonal variation related to changes in atmospheric density. The thresholds for the Arctic and Antarctic are very similar in magnitude and do not vary significantly from year-to-year. Typical mid-season values of the $\mathrm{R}_{532}$ threshold for the $450-550 \mathrm{~K}$ potential temperature layer are $2.60,1.82,1.51$, and 1.32 for horizontal smoothing scales of $5,15,45$, and $135 \mathrm{~km}$, respectively. Corresponding midseason values of the $\beta_{\text {perp }}$ thresholds are $3.62 \mathrm{e}-06 \mathrm{~km}^{-1} \mathrm{sr}^{-1}$, $3.53 \mathrm{e}-06 \mathrm{~km}^{-1} \mathrm{sr}^{-1}$, and $3.28 \mathrm{e}-06 \mathrm{~km}^{-1} \mathrm{sr}^{-1}$ for smoothing scales of 15,45 , and $135 \mathrm{~km}$.

\subsection{Ancillary meteorological products}

To aid in the analysis of the CALIPSO PSC observations, we incorporate ancillary meteorological data products including dynamic tropopause altitude and location relative to the vortex edge. For simplicity, we utilize existing Derived Meteorological Products (DMP) files produced for the Aura Microwave Limb Sounder (MLS) mission (Manney et al., 2007). Since Aura and CALIPSO are both in the Aqua satellite constellation (Stephens et al., 2002) with nearly identical orbits, the MLS and CALIPSO ground tracks are close to one another with a time offset of about eight minutes. The spatial separation varies with latitude from about $3 \mathrm{~km}$ at $80^{\circ}$ to $150 \mathrm{~km}$ at $50^{\circ}$. The DMP tropopause and vortex information are interpolated to the CALIPSO profile locations at $5-\mathrm{km}$ horizontal resolution. This information allows the cloud observations to be separated by their location with respect to the tropopause (stratospheric/tropospheric) and within the polar vortex. In addition, we interpolate the Aura MLS nitric acid 

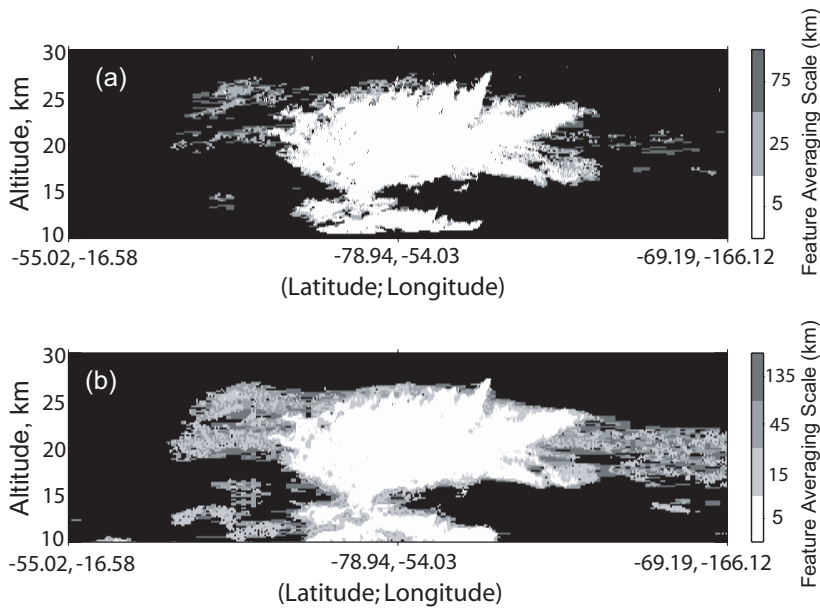

Fig. 2. PSC masks produced using the (a) v1 and (b) secondgeneration detection algorithms for an Antarctic cloud scene from 24 July 2006. The level of horizontal smoothing required to detect the cloud is indicated by the gray scale at the right of each image.

and water vapor gas phase mixing ratio measurements to the CALIPSO profile locations and include them in the PSC data product files to facilitate the calculation of PSC thermodynamic properties.

\subsection{Impact of algorithm enhancements on PSC detec- tion}

Figure 2 shows a comparison of the PSC masks produced for a sample cloud scene using the $\mathrm{v} 1$ and second-generation algorithms. The v1 algorithm (Fig. 2a) has no problem identifying the optically-thick regions of the PSC at the highest $(5-\mathrm{km})$ horizontal resolution. However, even with additional horizontal averaging to the coarser $(25-$ and $75-\mathrm{km})$ resolutions, it only detects small patches of optically-thin clouds scattered on either side of the main cloud shield. With the addition of the $\beta_{\text {perp }}$ channel, the second-generation algorithm (Fig. 2b) is much more sensitive to the presence of depolarizing, low-scattering ratio PSCs and detects large regions of these optically-thin clouds missed by the v1 algorithm.

Figure 3 shows a comparison of the daily areal coverage of PSCs in the Antarctic during the 2006 season as a function of altitude using the $\mathrm{v} 1$ and second-generation detection algorithms. The PSC areal coverage is estimated similar to Pitts et al. (2007) with some notable differences. The CALIOP observations are now binned into a $25 \times 25$ polar stereographic grid covering latitudes poleward of $50^{\circ}$. Each of the 625 grid boxes covers an area approximately $327 \mathrm{~km} \times 327 \mathrm{~km}$. The PSC frequency within each grid box as observed by CALIOP is assumed to be representative of the overall cloud fraction within that box. PSC areal coverage is calculated for each of four concentric regions within the grid, with each region being weighted in proportion to its relative area. This weighting accounts for both the uneven latitudinal sampling and the ten-
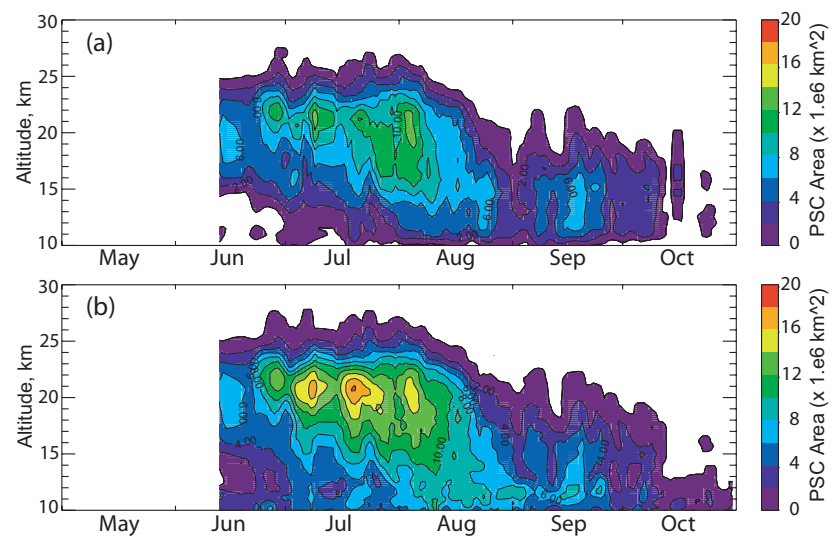

Fig. 3. Daily PSC areal coverage as a function of altitude for the 2006 Antarctic season calculated using the (a) v1 and (b) secondgeneration detection algorithms.

dency for PSCs to occur more frequently at higher latitudes. The total PSC areal coverage is then calculated by summing the cloud fractions from each of the four regions. We estimate the error in mapping the CALIOP PSC observations into areal coverage is generally less than $5 \%$, but may be larger when PSC coverage is limited such as very early and late in the Antarctic season and in the Arctic. Since we have defined our detection thresholds conservatively, the PSC areas likely represent a lower limit of true PSC coverage.

The impact of the $\beta_{\text {perp }}$ channel on PSC detection is clearly visible in Fig. 3 with approximately a $15 \%$ increase in the total PSC area detected by the second-generation algorithm versus the $\mathrm{v} 1$ algorithm. Most of this new cloud area is directly attributable to the additional depolarizing, lowscattering ratio PSCs now being detected.

\section{PSC composition discrimination}

Our goal in this version of the algorithm was to develop a single set of PSC composition classes to apply to all CALIOP data for the 2006-2008 Antarctic and 2006/2007-2007/2008 Arctic seasons. We adopted the coordinate system of aerosol (particle-only) depolarization ratio $\left(\delta_{\text {aerosol }}\right)$ vs. inverse scattering ratio $\left(1 / \mathrm{R}_{532}\right)$, following the approach used by Adriani et al. (2004) and Massoli et al. (2006) to analyze groundbased PSC data from McMurdo and Ny Ålesund, respectively. $\delta_{\text {aerosol }}$ is defined as the ratio of the perpendicular and parallel components of aerosol backscatter (Cairo et al., 1999), quantities that we calculated by subtracting estimates of the perpendicular and parallel components of molecular backscatter from the basic CALIOP measurements, assuming $\delta_{\text {molecular }}=0.00366$ (Hostetler et al., 2006). As a result of this estimation process and the inherently lower SNR of spaceborne measurements, the CALIOP data ensemble includes negative $\delta_{\text {aerosol values as well as anomalously large }}$ 


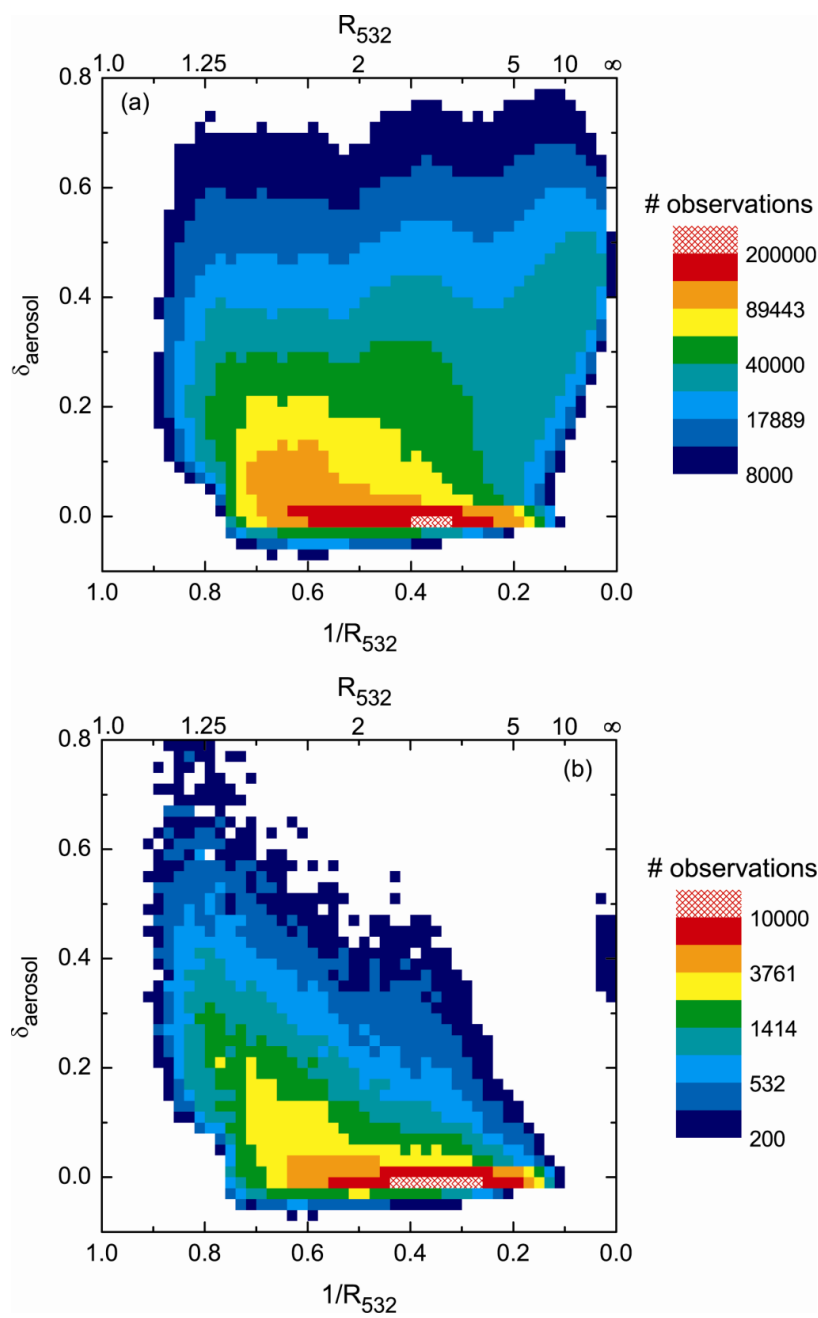

Fig. 4. 2-D histograms of CALIPSO PSC observations for the (a) 2006-2008 Antarctic and (b) 2006-2007/2007-2008 Arctic seasons.

values, especially as $1 / \mathrm{R}_{532}$ approaches 1.0 . Figure 4 shows composite 2-D histograms of CALIOP PSC data for (a) the three Antarctic winters and (b) the two Arctic winters with a bin size of 0.02 by 0.02 , and color scales indicate the total number of data pairs $\left(1 / \mathrm{R}_{532}, \delta_{\text {aerosol }}\right)$ falling within each bin. The axis ranges in Fig. 4 have been limited for presentation purposes; about $14 \%$ overall of CALIOP PSC data points fall outside these limits. Note that our horizontal scale is $1 / \mathrm{R}_{532}$, the converse of that used by Adriani et al. (2004) and Massoli et al. (2006).

Figure 4 shows that many more PSCs have been observed by CALIOP in the Antarctic than in the Arctic, as expected from published climatologies. The highest concentration of points in both histograms in Fig. 4 is the horizontal cluster around $\delta_{\text {aerosol }}=0$, which is similar to the set of ground-based observations classified as "Liquid" by Adriani et al. (2004) and Massoli et al. (2006). Other regions of the CALIOP PSC histograms differ considerably from the comparative McMurdo and $\mathrm{Ny}$ Ålesund plots. In particular, relatively more CALIOP PSC data points lie in the range $\left[0.4<1 / \mathrm{R}_{532}<0.8,0.03<\delta_{\text {aerosol }}<0.2\right]$ observations classified as "Mixed" or "MixA" in the ground-based data sets, as well as at very small values of $1 / R_{532}$, observations classified as "ice" in the ground-based data sets. Also, the general shape of the CALIPSO data contours does not conform well to the box-like PSC composition classes used for the groundbased PSC observations. Therefore, we chose to define new CALIPSO-based composition classes guided by the optical model calculations described in the next section.

\subsection{Optical calculations for liquid-solid PSC mixtures}

Our calculations assumed a single STS particle population, as well as plausible mixtures of STS and primarily low number density NAT or ice particles. The range in particle size and number density was based on in situ observations (Fahey et al., 2001; Northway et al., 2002; Dye et al., 1992). In all cases, total particle number density ( $\mathrm{N}_{\mathrm{TOTAL}}$ ) was fixed at $10 \mathrm{~cm}^{-3}$, and condensed particle volumes were constrained to not exceed equilibrium thermodynamic values prescribed by Carslaw et al. (1995) for STS, Hanson and Mauersberger (1988) for NAT, and the Goff-Gratch formulation (List, 1984) for ice. We assumed $50 \mathrm{hPa}$ atmospheric pressure and nominal vapor mixing ratios of $10 \mathrm{ppbv} \mathrm{HNO}_{3}$ and $5 \mathrm{ppmv}$ $\mathrm{H}_{2} \mathrm{O}$; for these conditions, $T_{\mathrm{NAT}} \cong 195.7 \mathrm{~K}, T_{\text {ice }} \cong 188.5 \mathrm{~K}$, and $T_{\mathrm{STS}}$ (the temperature at which STS volume starts to increase markedly) $\cong 192 \mathrm{~K}$. Particle optical properties were calculated using the T-matrix approach of Mishchenko and Travis (1998), with fixed refractive indices of 1.43 for STS, 1.50 for NAT, and 1.308 for ice. The imaginary parts of the refractive indices are assumed to be 0.0 for all constituents. We assumed STS particles to be spheres (aspect ratio $=1.0$ ) and assumed both NAT (Larsen et al., 2004) and ice particles to be oblate spheroids with an aspect ratio of 1.2. The calculated depolarization ratio for non-spherical particles is sensitive to aspect ratio, and we intend to explore this sensitivity with future enhancements of the CALIPSO PSC algorithm.

Details of our notional PSC particle scenarios are as follows:

(1) STS only: Lognormal particle size distribution with $\sigma=1.6 ; \mathrm{N}_{\mathrm{STS}}=10 \mathrm{~cm}^{-3}$; mode radius derived from the equilibrium condensed STS volume for $196 \mathrm{~K}>T>T_{\text {ice }}-3 \mathrm{~K}$.

(2) STS-NAT mixtures: Inverse power law NAT size distribution with fixed effective variance $=0.1$ (Liu and Mishchenko, 2001); $\{\mathrm{a}, \mathrm{b}\} \quad \mathrm{N}_{\mathrm{NAT}}=\left[10^{-4}, 10^{-3}\right] \mathrm{cm}^{-3}$ and $r_{\text {eff,NAT }}=[1,3,5,7] \mu \mathrm{m} ; \quad\{c\} \quad \mathrm{N}_{\mathrm{NAT}}=10^{-2} \mathrm{~cm}^{-3}$ and $\mathrm{r}_{\mathrm{eff}, \mathrm{NAT}}=[1,2,3,4] \mu \mathrm{m} ; \quad\{\mathrm{d}\} \quad \mathrm{N}_{\mathrm{NAT}}=10^{-1} \mathrm{~cm}^{-3} \quad$ and $\mathrm{r}_{\text {eff, NAT }}=[0.5,1.0,1.5] \mu \mathrm{m}$. STS treated as in scenario (1), but with $\mathrm{N}_{\mathrm{STS}}=\mathrm{N}_{\mathrm{TOTAL}}-\mathrm{N}_{\mathrm{NAT}}$, and STS equilibrium conditions adjusted to account for $\mathrm{HNO}_{3}$ in co-existing NAT particles. 

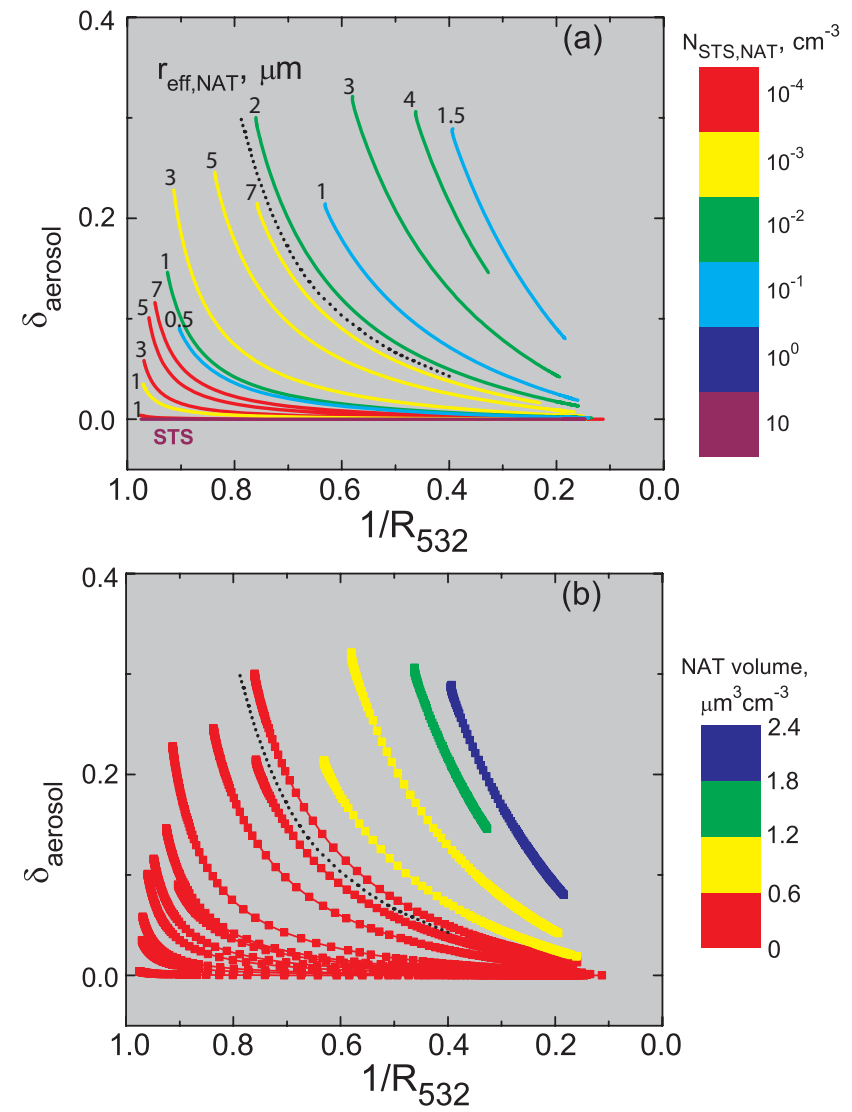

Fig. 5. Theoretical results for STS and STS-NAT mixtures. Color scale $=$ (a) Particle number density; (b) NAT particle volume.

(3) STS-ice mixtures: Inverse power law ice size distribution, with fixed effective variance $=0.1$; \{a $\mathrm{N}_{\text {ice }}=\left[10^{-2}\right] \mathrm{cm}^{-3}$ and $\mathrm{r}_{\text {eff, ice }}=[1,7] \mu \mathrm{m} ; \quad\{\mathrm{b}\}$ $\mathrm{N}_{\text {ice }}=\left[10^{-1}\right] \mathrm{cm}^{-3} \quad$ and $\quad \mathrm{r}_{\text {eff, ice }}=[1,3,5,7] \mu \mathrm{m} ; \quad\{\mathrm{c}\}$ $\mathrm{N}_{\text {ice }}=1 \mathrm{~cm}^{-3}, \quad \mathrm{r}_{\text {eff, } \text {,ice }}=[1,2,3] \mu \mathrm{m} ; \quad\{\mathrm{d}\} \quad \mathrm{N}_{\text {ice }}=10 \mathrm{~cm}^{-3}$ (wave cloud) and $r_{\text {eff,ice }}=[0.5,1.0,1.5] \mu \mathrm{m}$. STS treated as in scenario (1), with $\mathrm{N}_{\mathrm{STS}}$ reduced to $\left(\mathrm{N}_{\mathrm{TOTAL}}-\mathrm{N}_{\mathrm{ice}}\right)$ at temperatures below the frost point.

Figure 5 shows our theoretical results for STS and STSNAT mixtures, color-coded by particle number density in Fig. 5a and by NAT particle volume in Fig. 5b. Along each curve, temperature decreases implicitly from its maximum at the left to $185.5 \mathrm{~K}$ at the right. For the STS line (along $\left.\delta_{\text {aerosol }}=0\right), T_{\max }=196 \mathrm{~K}$; for each STS-NAT mixture curve, $T_{\max }$ is the highest temperature below $T_{\mathrm{NAT}}$ where the condensed NAT volume $\leq$ equilibrium NAT volume. The ensemble of computed STS-NAT mixture curves reasonably mimics the shape of the CALIPSO PSC data contours in the range $0.8>1 / R_{532}>0.2$. The mixture curves all converge toward the STS line at lower temperatures $\left(T<T_{\mathrm{STS}}\right)$ because the optical signature of the NAT particles is being masked by the more numerous (and growing) STS particles, as has been noted in a number of previous studies (e.g., Shibata et

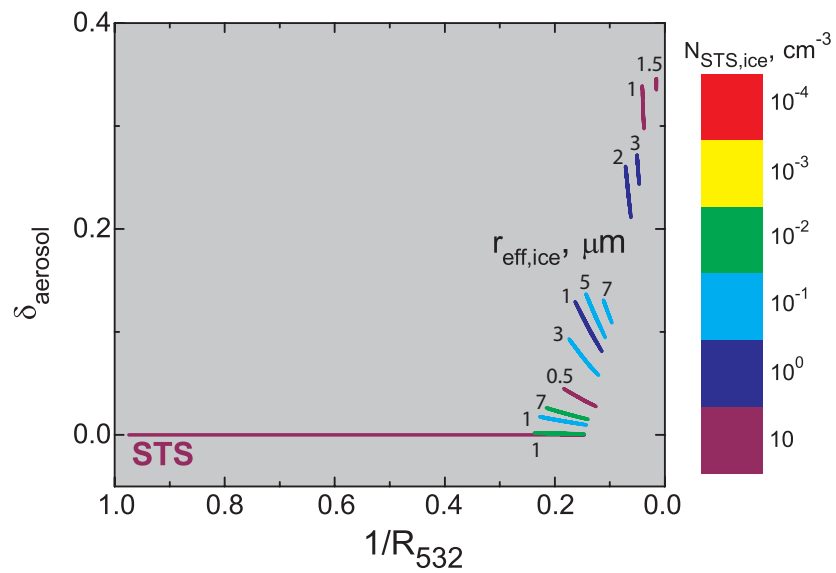

Fig. 6. Theoretical results for STS and mixtures of STS with ice. Color scale $=$ particle number density.

al., 1999; Larsen et al., 2004). The black dotted curve has been added to highlight the fact that the computed results are separated reasonably well according to NAT number density and/or volume. Mixtures with lower NAT number density or lower NAT volume tend to fall below/to the left of the dotted curve, while those with higher number density and higher volume tend to lie above/to the right of the curve.

Figure 6 shows analogous computed results for STS and STS-ice mixtures. Again, temperature decreases implicitly along each curve from left (top) to right (bottom). $T_{\max }$ is the highest temperature below $T_{\text {ice }}$ where condensed ice volume $\leq$ equilibrium ice volume, and $T_{\min }=T=185.5 \mathrm{~K}$. Note that the ensemble of computed STS-ice mixture curves reasonably mimics the "arm" of the CALIPSO data histogram extending upward toward larger values of $\delta_{\text {aerosol }}$ over the range $0.2>1 / R_{532}>0$.

\subsection{CALIPSO PSC composition classes}

Based on our theoretical calculations, we made the arbitrary choice of four composition classes for CALIOP PSC data: Mix 1, Mix 2, ice, and STS. The domains of these composition classes are shown in Fig. 7, which is a composite 2D histogram for all five PSC seasons observed by CALIOP to date. Note that since CALIOP data are noisy, there is some uncertainty in assigning an observation to one particular composition class, especially if the data point lies near the boundary between two classes. As discussed in the previous section, we defined two classes of mixed STS-NAT clouds, with Mix 1 and Mix 2 denoting lower and higher NAT number density/volume, respectively. We specified the boundary between these two classes by an empirical curve overlying the computed points for $\left[\mathrm{N}_{\mathrm{NAT}}=10^{-3} \mathrm{~cm}^{-3}, \mathrm{r}_{\mathrm{eff}, \mathrm{NAT}}=7 \mu \mathrm{m}\right]$ in the range $0.8 \leq 1 / \mathrm{R}_{532} \leq 0.2$. We also assigned to the Mix 1 class all PSCs with $1 / R_{532}>0.8$, including those detected via enhancements in $\beta_{\perp}$ but which fall outside the limits of the histograms shown in Fig. 4. A comparison of Figs. 5 and 


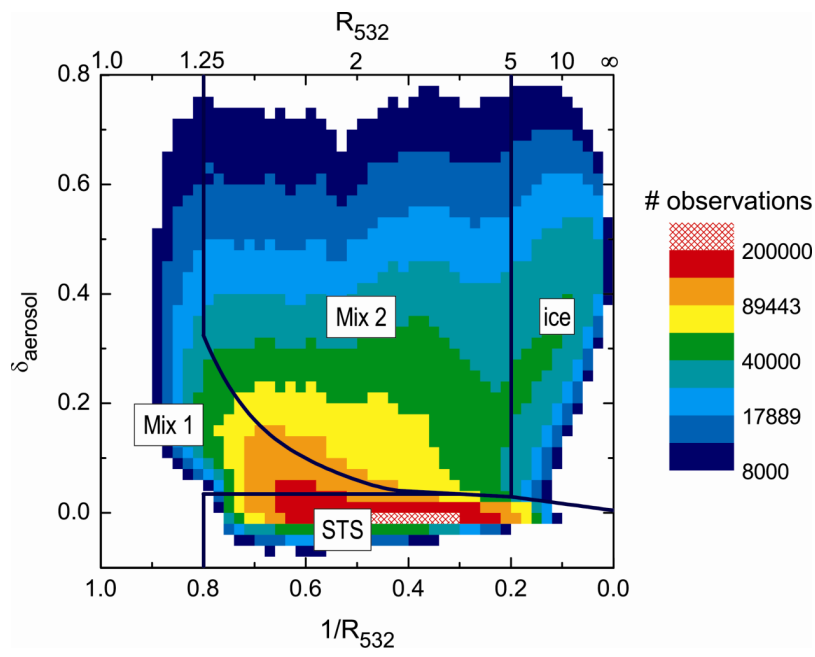

Fig. 7. Composite histogram of CALIPSO PSC observations for Antarctic 2006-2008 seasons and Arctic 2006/2007-2007/2008 seasons, with new composition classification boundaries.

6 suggested $1 / R_{532}=0.2$ as a good choice for the boundary in backscatter between the Mix 2 and ice classes. Note that even though observations are classified as "ice," our calculations suggest they are very likely STS-ice mixtures instead, except in the case of wave clouds, where all STS droplets might be expected to freeze and serve as ice nuclei.

To be consistent with previous studies, our fourth composition class is named STS, even though our calculations indicate this class also includes STS-solid mixtures whose optical signature is dominated by the more numerous liquid droplets. The upper bound on $\delta_{\text {aerosol }}$ for STS has generally been set as a constant in the past (e.g., 0.03 in the studies by Adriani et al., 2004 and Massoli et al., 2006). However, the fact that contours in the CALIPSO data histogram broaden

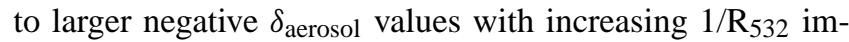
plies that the uncertainty in $\delta_{\text {aerosol }}$ varies along the ordinate. Therefore, we felt it was prudent to allow the upper bound in $\delta_{\text {aerosol }}$ for our STS class to vary with $1 / \mathrm{R}_{532}$, and we defined it using a probability distribution function (PDF) approach patterned after the CALIPSO cloud-aerosol discrimination algorithm (Liu et al.. 2004).

The 1-D CALIPSO PSC $\delta_{\text {aerosol }}$ PDF can be visualized as a vertical "slice" through the $2-\mathrm{D}\left(1 / \mathrm{R}_{532}, \delta_{\text {aerosol }}\right)$ histogram at some value of $1 / R_{532}$. We formed a series of such PDFs (Fig. 8) from a 2-D histogram analogous to Fig. 4, but with higher resolution (0.002) $\delta_{\text {aerosol }}$ bins and compensating lower resolution (0.05) $1 / \mathrm{R}_{532}$ bins. Each measured PDF (including those for intermediate $1 / \mathrm{R}_{532}$ bins not shown here) has a mode near $\delta_{\text {aerosol }}=0$, which we interpret as the signature of the STS PSC class. We assume that for a given $1 / R_{532}$ bin, the measured PDF is the sum of overlapping PDFs for the STS class and a "residual" (STS/solid mixture) class with higher $\delta_{\text {aerosol }}$ values, an analogy to the overlapping cloud and aerosol backscatter PDFs used by Liu et

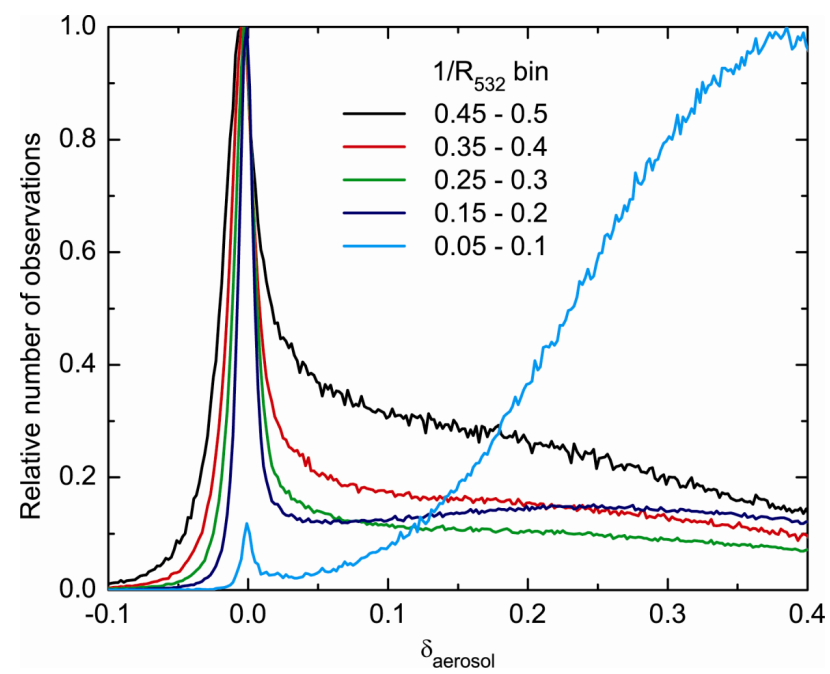

Fig. 8. Normalized CALIPSO PSC $\delta_{\text {aerosol }}$ probability distributions functions (PDFs) for a series of $1 / R_{532}$ bins.

al. (2004). We further assume that the STS PDF is symmetric about its mode near $\delta_{\text {aerosol }}=0$, i.e. we set the number of observations in bins to the right of the mode to be equal to the measured number in the analogous bins to the left of the mode. This is similar to the approach used by Fromm et al. (2003) to define a baseline aerosol-only extinction PDF for identifying PSCs in solar occultation data. The "residual" PDF is formed by subtracting the STS PDF from the measured PDF, and we can then calculate a confidence function after Liu et al. (2004) that quantifies the differential occurrence probability of the STS and residual STS/solid mixture classes. We chose to set the STS upper bound at the value of $\delta_{\text {aerosol }}$ for which the confidence function $=0.5$, i.e. the value above which $>75 \%$ of the observations fall in the residual mixture class. An example of this process is shown in Fig. 9 for the bin $0.25<1 / R_{532}<0.3$. Values of the STS upper bound calculated using this procedure varied between $0.03-0.04$ over the range $0.8>1 / R_{532}>0.3$, were near 0.03 in the range $0.3>1 / R_{532}>0.2$, and decreased monotonically to a value near 0.005 for the $0.05>1 / R_{532}>0$ bin. For simplicity in our algorithm, the STS upper bound is constant $=0.035$ for $0.8>1 / R_{532}>0.4$, then decreases linearly to 0.03 at $1 / R_{532}<0.3$, and then decreases linearly again to 0.005 at $1 / \mathrm{R}_{532}=0$.

Figure 10 shows the same PSC image as from Fig. 2, but with the composition definitions applied to the measurements. The dense core of the expansive cloud system is dominated by water ice particles. Surrounding the central ice cloud are extensive regions of mixtures containing NAT particles. On the lower right side of the ice cloud is a small region dominated by liquid STS droplets. An underlying cirrus deck is also visible. Overall, as we typically observe, the different compositions exhibit good spatial coherence. 


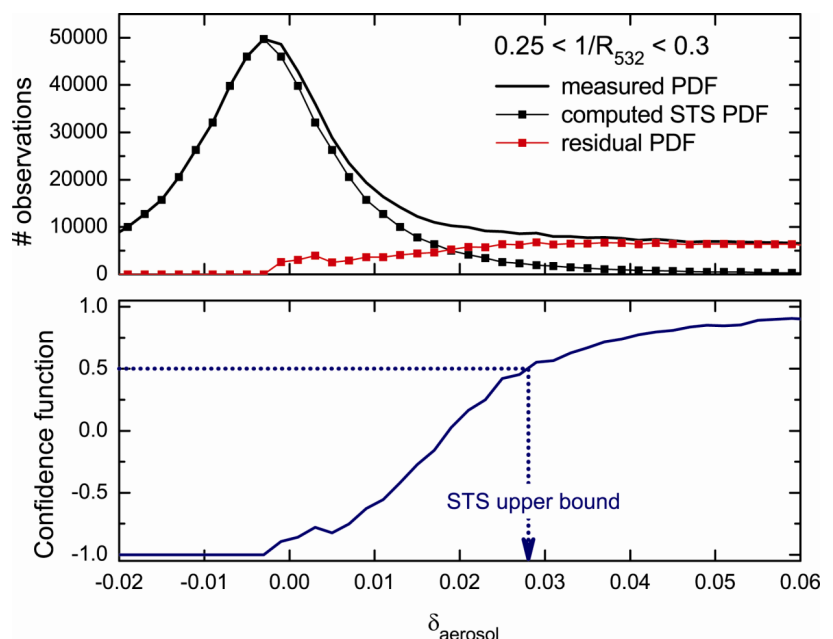

Fig. 9. Illustration of procedure used to define the upper bound in $\delta_{\text {aerosol }}$ for the STS PSC composition class.

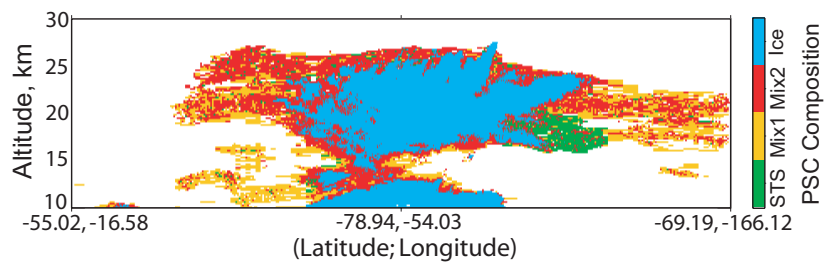

Fig. 10. PSC composition discrimination for the cloud scene from 24 July 2006 (same scene as shown in Fig. 2).

An extensive comparison between PSC observations by CALIPSO and the infrared limb emission sounder MIPAS (Michelson Interferometer for Passive Atmospheric Sounding) on the Envisat spacecraft has recently been performed (Höpfner et al., 2009). Both instruments detected PSCs in about $90 \%$ of Antarctic coincidences in 2006-2007, and in 60-70\% of Arctic coincidences in the 2006/2007-2007/2008 winters. There was also a high degree of consistency between PSC composition derived from the two instruments. Of particular note is that for PSCs in which the spectral signature of NAT was detected by MIPAS, about $90 \%$ of all coincident CALIPSO data were identified as Mix 1 or Mix 2. Furthermore, the distinction between the CALIPSO Mix 1 and Mix 2 classes was found to be well correlated with MIPAS cloud indices in terms of particle volume densities.

\section{Data analyses}

Using the second-generation detection algorithm and composition classification scheme, we have produced a new database of CALIPSO PSC observations covering to date three seasons in the Antarctic and two seasons in the Arctic. In this section we examine this database and illustrate its
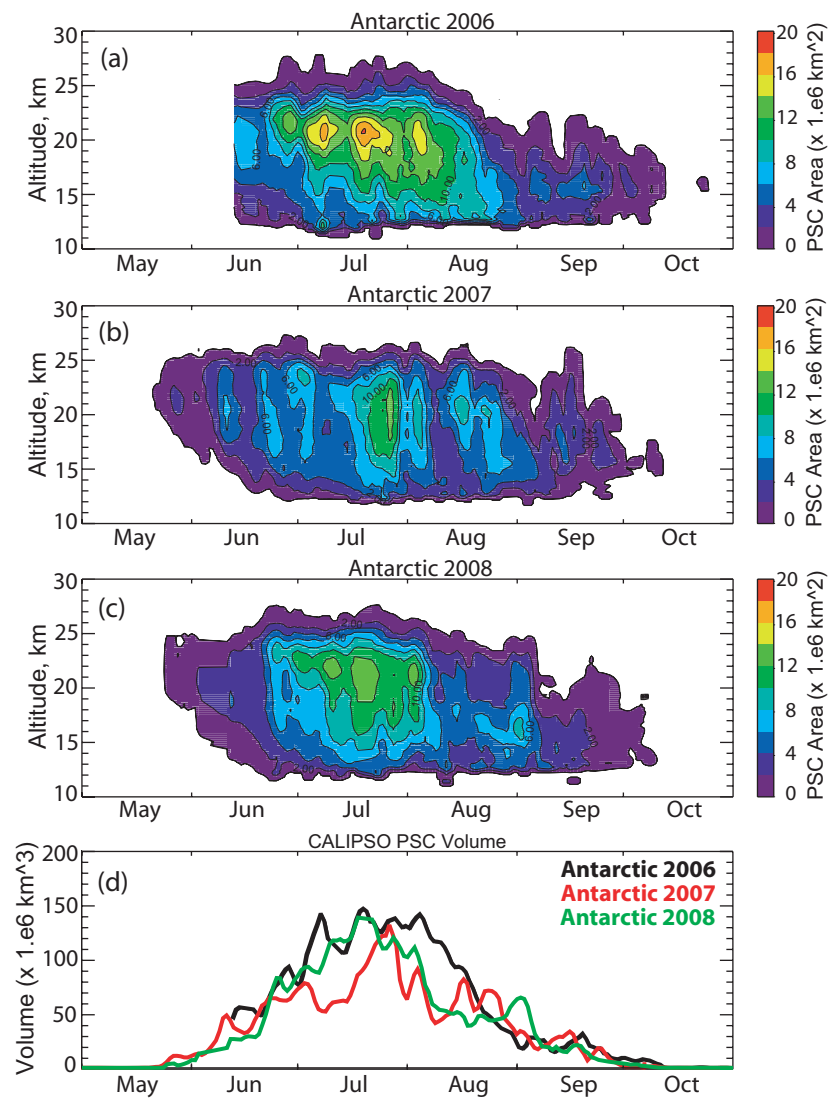

Fig. 11. Daily time series of CALIPSO PSC area as a function of altitude for the (a) 2006, (b) 2007, and (c) 2008 Antarctic seasons. The total volume PSC coverage for all three seasons is shown in panel (d).

unique attributes for PSC composition studies. To avoid cirrus contamination, we have limited these analyses to include only observations $\geq 4 \mathrm{~km}$ above the local tropopause. With CALIPSO's extensive measurement coverage over the polar regions, it is possible to characterize PSCs over large spatial regions on short time scales. For example, time series of the daily areal coverage of PSCs over the Antarctic region during the 2006-2008 seasons are shown in Fig. 11a-c. The 2006 Antarctic winter vortex was one of the coldest and largest in areal extent on record and this led to extensive PSC formation with maximum cloud coverage in July and August (note that CALIOP began routine data acquisition in mid-June 2006). The 2007 Antarctic winter vortex was not as cold or expansive as in 2006 and this is reflected in the lower overall PSC occurrence. The 2008 winter vortex was colder than in 2007 , but not as cold as the record 2006 winter. Thus, as expected, the observed PSC areal coverage in 2008 was between the 2006 and 2007 values. The differences between these three years illustrate the substantial year-to-year variability of PSC occurrence in the Antarctic with cloud formation directly linked to the dynamics (i.e., thermal structure, size, stability) 

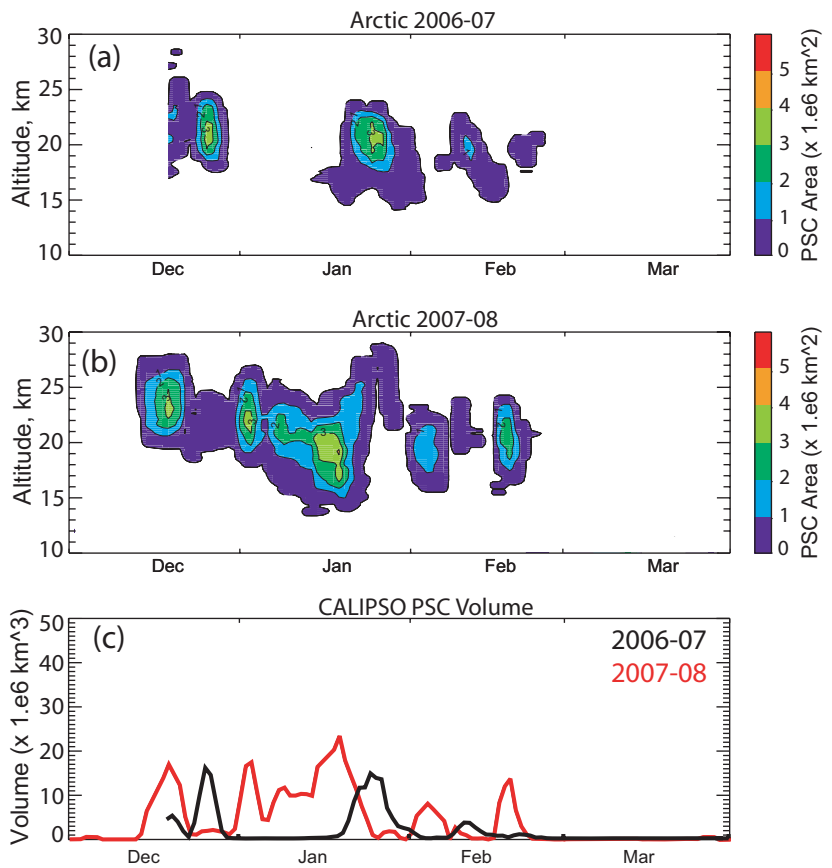

Fig. 12. Daily time series of CALIPSO PSC area as a function of altitude for the (a) 2006-2007 and (b) 2007-2008 Arctic seasons. The total volume PSC coverage for both years is shown in panel (c).

of the vortex. By integrating the daily PSC areas vertically, a time series of the total atmospheric volume covered by PSCs $\left(\mathrm{V}_{\mathrm{PSC}}\right)$ is produced. This is a unique capability of CALIPSO and provides the first observation-based estimates of $V_{P S C}$. $\mathrm{V}_{\text {PSC }}$ for all three Antarctic seasons is shown in Fig. 11d. In terms of season totals, the 2007 PSC volume was about $20 \%$ less than for the record 2006 season with most of this difference occurring in July and August. The 2008 season PSC volume started on par with the record 2006 season, but PSC coverage relative to 2006 dropped off significantly in August and the overall 2008 PSC volume ended about $16 \%$ lower than in 2006.

The PSC areal coverage for the 2006-2007 and 20072008 Arctic seasons is shown in Fig. 12a-b. The Arctic polar vortex is less stable and warmer than its Antarctic counterpart and, as a result, PSC occurrence in the Arctic is much more episodic in nature and restricted to significantly smaller spatial scales than in the Antarctic. PSC coverage during the 2006-2007 Arctic season was very sparse with only a few sporadic periods of PSC activity (note that CALIOP did not acquire data during the first half of December 2006). The 2007-2008 Arctic season was more active with PSCs observed during the latter half of December and throughout most of January, with another late burst of activity in the latter half of February. The $V_{\text {PSC }}$ for both Arctic years is shown in Fig. 12c. The coverage of PSCs in the Arctic is at least a factor of 5 less than in the Antarctic.
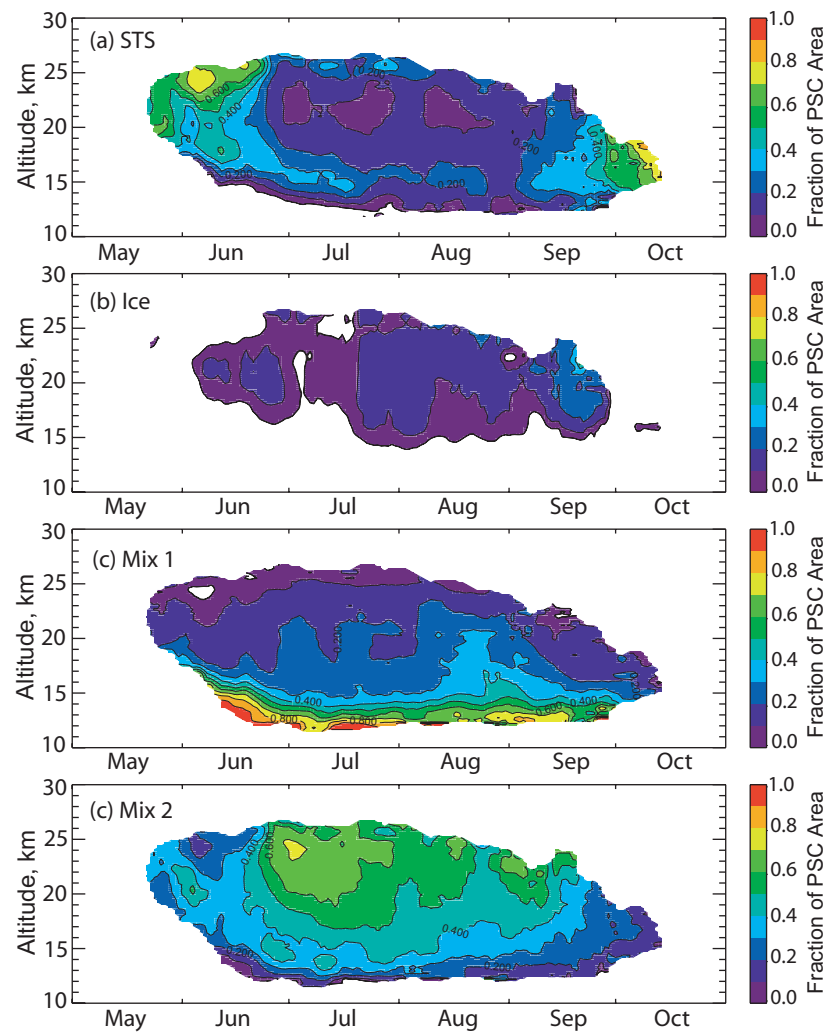

Fig. 13. Time series of three-year mean Antarctic PSC areal coverage for each of the four composition classes: (a) STS, (b) ice, (c) Mix 1, and (d) Mix 2. The values have been normalized by the total PSC area to show the relative coverage of each composition class as indicated by the color bars.

In addition to capturing the seasonal evolution of PSC occurrence, the CALIPSO database also provides the opportunity to explore the seasonal evolution of PSCs by composition class. To illustrate this capability, we combined the 2006-2008 Antarctic data to produce seasonal time series of the three-year mean PSC areal coverage for each of the four composition classes defined in Sect. 3. The time series are shown in Fig. 13a-d and have been normalized by the total PSC areal coverage. STS clouds (Fig. 13a) first appear in late May at altitudes from $20-25 \mathrm{~km}$, and STS is the most prevalent composition class early in the season. STSNAT mixtures increase dramatically by mid-June, and Mix 1 and Mix 2 are the predominant composition classes observed throughout the stratosphere in July and August. The distribution of the Mix 1 and Mix 2 observations has a striking vertical dependence. Below $15 \mathrm{~km}$, Mix 1 PSCs are by far the most frequently observed composition class, approaching $100 \%$ of the total observed PSC area at altitudes nearest the tropopause. Above $15 \mathrm{~km}$, the fraction of Mix 1 observations decreases sharply and Mix 2 becomes the predominant composition class. By mid-September, STS once again becomes the most frequently observed composition class, but 

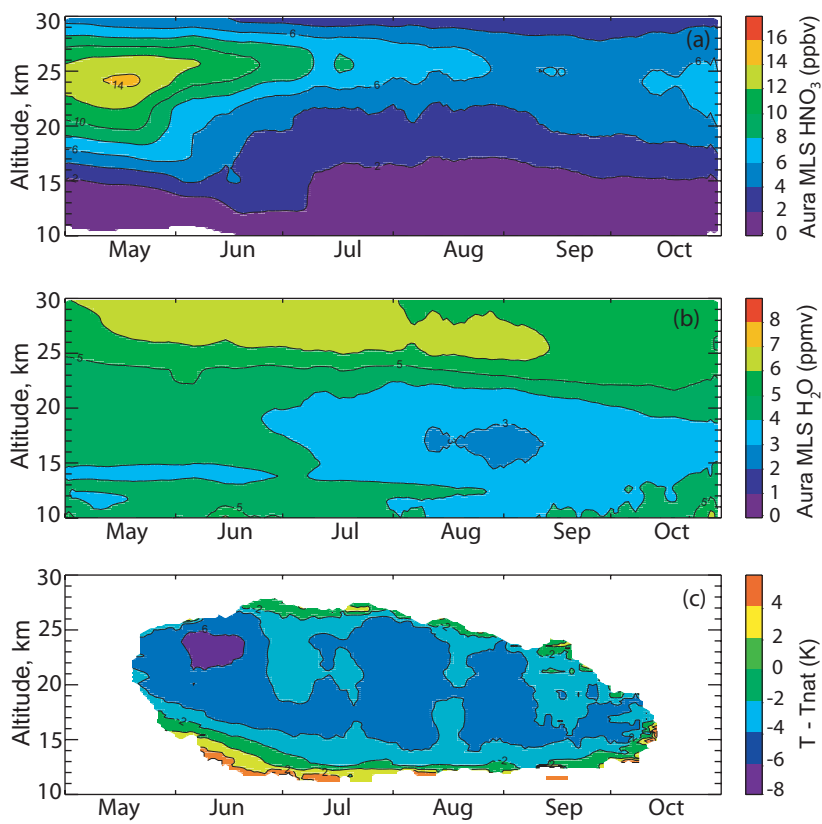

Fig. 14. Time series of three-year mean Aura $\mathrm{MLS}$ (a) $\mathrm{HNO}_{3}$ and (b) $\mathrm{H}_{2} \mathrm{O}$ mixing ratio observations in PSC-free regions of the Antarctic vortex. The corresponding vortex-averaged differences $(\Delta T)$ between ambient temperature at CALIPSO PSC locations and

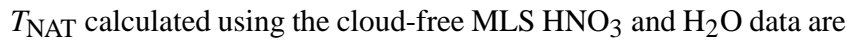
shown in panel (c).

the maximum occurs at much lower altitudes $(15-17 \mathrm{~km})$ than the early season STS maximum. The re-emergence of STS late in the season warrants further investigation. Ice PSCs (Fig. 13b) are more episodic in nature and are the least frequent composition class observed throughout the season with coverage rarely exceeding $20 \%$ of the total PSC area. The majority of ice clouds are observed near the Antarctic Peninsula or near the Transantarctic Mountains, suggesting that orographic waves are a primary formation mechanism. Although it is difficult to compare our results directly with previous findings due to differences in terminology and composition definitions, the seasonal evolution of Antarctic PSC composition observed by CALIPSO agrees qualitatively with the findings of Adriani et al. (2004) based on multiyear observations at McMurdo Station.

Time series of three-year average Aura MLS Antarctic $\mathrm{HNO}_{3}$ and $\mathrm{H}_{2} \mathrm{O}$ mixing ratio data in PSC-free regions are shown in Fig. 14a-b. These correspond to the PSC composition figures shown in Fig. 13 and were produced by interpolating MLS data to the CALIPSO ground tracks (as described in Sect. 2.4), but excluding data points where PSCs were observed by CALIPSO. These time series are not affected by local vapor uptake on PSCs and hence should provide a reasonable estimate of the total available $\mathrm{HNO}_{3}$ and $\mathrm{H}_{2} \mathrm{O}$, provided that the air within the vortex is well-mixed. Fig. 14c shows the corresponding vortex-average difference $(\Delta T)$ be- tween ambient temperature (from GEOS5) at CALIPSO PSC locations and $T_{\mathrm{NAT}}$, with the latter computed using the cloudfree MLS $\mathrm{HNO}_{3}$ and $\mathrm{H}_{2} \mathrm{O}$ values in the Hanson and Mauersberger (1988) relationship.

There is a high degree of consistency between the MLS observations and our inferences of PSC composition. Early in the season when gas-phase $\mathrm{HNO}_{3}$ and $\mathrm{H}_{2} \mathrm{O}$ are relatively abundant, STS clouds are the predominant composition class, and occur at the largest negative values of $\Delta T$ ( $\sim 6 \mathrm{~K}$ below $\left.T_{\mathrm{NAT}}\right)$, a result consistent with many earlier studies (e.g., Weisser et al., 2006). NAT mixtures become the most common compositions in mid-June, and are found at less negative values of $\Delta \mathrm{T}\left(\sim 2-5 \mathrm{~K}\right.$ below $\left.T_{\mathrm{NAT}}\right)$, where STS droplet growth is limited and the presence of NAT particles is not totally masked by the more numerous STS droplets. The total gas-phase $\mathrm{HNO}_{3}$ abundance also begins to decrease significantly in June, suggesting that NAT plays a pivotal role in the denitrification of the Antarctic vortex. The observed decrease in MLS total gas-phase $\mathrm{H}_{2} \mathrm{O}$ occurs primarily in July and August, and is reasonably well correlated with the maximum in areal extent of ice PSCs. Mix 1 PSCs are the predominant composition when temperatures are very close to $T_{\mathrm{NAT}}$, particularly in the lower stratosphere. STS droplet growth is negligible in this temperature range, increasing the likelihood of detecting the presence of NAT particles in very low number densities or volumes. Late in the season, the total gas-phase $\mathrm{HNO}_{3}$ and $\mathrm{H}_{2} \mathrm{O}$ abundances begin to recover and the predominant PSC composition class again becomes STS. While these are broad-brush depictions, they do suggest that our ultimate goal to better understand PSC microphysical processes and stratospheric denitrification through analysis of CALIPSO and ancillary (e.g., MLS) data is attainable.

\section{Summary and conclusions}

We have implemented our second-generation CALIPSO PSC detection algorithm which incorporates a number of enhancements compared to the v1 algorithm. Most noteworthy is the addition of the perpendicular backscatter measurements to the detection process which significantly increases our ability to detect the presence of tenuous PSCs containing low number densities of solid NAT particles. Overall, the second-generation algorithm detects approximately $15 \%$ more PSC area than the $\mathrm{v} 1$ algorithm with most of this additional area attributed to these optically-thin NAT cloud mixtures. Although these low number density NAT mixtures would have a minimal impact on chlorine activation due to their relatively small particle surface area, they may play a significant role in denitrification and therefore are an important component of our PSC detection.

We have also developed an algorithm to partition the CALIPSO PSC observations into four composition classes based on the measured ensemble aerosol depolarization and inverse scattering ratio. We defined two classes of mixed 
STS-NAT clouds, with Mix 1 and Mix 2 denoting lower and higher NAT number density/volume, respectively. A third class is named ice, although these are probably STS-ice mixtures except in the case of wave clouds. To be consistent with earlier works, our fourth composition class is named STS (i.e., liquid), but this class probably includes STS-solid mixtures as well, under conditions where the ensemble optical signature is dominated by the more numerous liquid droplets.

We applied the detection and composition classification algorithms to the CALIOP data from three Antarctic and two Arctic seasons to examine the seasonal evolution of PSCs. These analyses show substantial year-to-year changes in PSC areal coverage and clearly depict the well-know contrast between the Antarctic and Arctic. A more in-depth analysis of the Antarctic data revealed unique seasonal and altitudinal variations in inferred PSC composition. STS is the most frequent composition class detected early and late in the Antarctic season. STS-NAT mixtures (Mix 1 and Mix 2) are the predominant composition classes observed throughout the stratosphere from mid-June until mid-September, but are distributed preferentially in altitude. Mix 1 is the predominant composition class in the lower stratosphere $(<15 \mathrm{~km})$, while Mix 2 is the predominant class at higher altitudes. Although ice PSCs are the least common composition class observed in the Antarctic, the majority of the observations occur near the Antarctic Peninsula or the Transantarctic Mountains suggesting that orographic waves are a primary formation mechanism.

The seasonal evolution of inferred PSC composition is consistent with MLS observations of gas-phase $\mathrm{HNO}_{3}$ and $\mathrm{H}_{2} \mathrm{O}$. There is good correlation between the significant increase in observations of NAT mixtures and decrease in total gas-phase $\mathrm{HNO}_{3}$ abundance in June. There is also reasonable correspondence between the maximum in areal extent of ice PSCs and the decrease in MLS total gas-phase $\mathrm{H}_{2} \mathrm{O}$ that occurs primarily in July and August. These suggest that NAT/ice PSCs play an important role in the denitrification/dehydration of the Antarctic vortex. As a result, we believe that our goal to better understand PSC microphysical processes and stratospheric denitrification through analysis of CALIPSO and ancillary (e.g., MLS) data is attainable.

Acknowledgements. The Derived Meteorological Products for Aura MLS were calculated and provided by G. Manney and W. Daffer with support from the MLS team at NASA's Jet Propulsion Laboratory, California Institute of Technology. The Aura MLS gas species data were provided courtesy of the MLS team and obtained through the Aura MLS website (http://mls.jpl.nasa.gov/index-eos-mls.php). IDL routines for calculating PSC thermodynamic properties were obtained from the GATS Scientific Software website (http://gwest.gats-inc.com/software/software_page.html). We would like to thank N. Larsen for his helpful suggestions and comments related to this work. We would also like to thank Dr. Hal Maring, NASA Radiation Sciences Program manager, and the NASA HQ Earth Science Division for continued support of this research. Support for L. Poole is provided under NASA contract NNL07AA00C.

Edited by: J. Quaas

\section{References}

Adriani, A., Massoli, P., Di Donfrancesco, G., Cairo, F., and Moriconi, M. L., and Snels, M.: Climatology of polar stratospheric clouds based on lidar observations from 1993 to 2001 over McMurdo Station, Antarctic, J. Geophys. Res., 109, D24211, doi:10.1029/2004JD004800, 2004.

Biele, J., Tsias, A., Luo, B. P., Carslaw, K. S., Neuber, R., Beyerle, G., and Peter, T.: Nonequilibrium coexistence of solid and liquid particles in Arctic stratospheric clouds, J. Geophys. Res., 106, 22991-23007, 2001.

Browell, E. V., Butler, C. F., Ismail, S., Robinette, P. A., Carter, A. F., Higdon, N. S., Toon, O. B., Schoeberl, M. R., and Tuck, A. F.: Airborne lidar observations in the wintertime Arctic stratosphere: polar stratospheric clouds, Geophys. Res. Lett., 17, 385388, 1990 .

Cairo, F., Di Donfrancesco, G., Adriani, A., Pulvirenti, L., and Fierli, F.: Comparison of various linear depolarization parameters measured by lidar, Appl. Opt., 38, 4425-4432, 1999.

Carslaw, K. S., Luo, B. P., Peter, T.: An analytic expression for the composition of aqueous $\mathrm{HNO}_{3}-\mathrm{H}_{2} \mathrm{SO}_{4}$ stratospheric aerosols including gas phase removal of $\mathrm{HNO}_{3}$, Geophys. Res. Lett., 22, 1877-1880, 1995.

Dye, J. E., Baumgardner, D., Gandrud, B. W., Kawa, S. R., Kelly, K. K., Loewenstein, M., Ferry, G. V., Chan, K. R., and Gary, B. L.: Particle size distributions in Arctic polar stratospheric clouds, growth and freezing of sulfuric acid droplets, and implications for cloud formation, J. Geophys. Res., 97(D8), 8015-8034, 1992.

Fahey, D. W., Gao, R. S., Carslaw, K. S., Kettleborough, J., Popp, P. J., Northway, M. J., et al.: The detection of large $\mathrm{HNO}_{3}$ containing particles in the winter Arctic stratosphere, Science, 291, 1026-1031, 2001.

Fromm, M. D., Alfred, J. M.., and Pitts, M.: A unified longterm, high-latitude stratospheric aerosol and cloud database using SAM II, SAGE II, and POAM II/III data: Algorithm description, database definition and climatology, J. Geophys. Res., 108(D12), 4366, doi:10.1029/2002JD002772, 2003.

Gobbi, G. P., Di Donfrancesco, G., and Adriani, A.: Physical properties of stratospheric clouds during the Antarctic winter of 1995, J. Geophys. Res., 103, 10859-10873, 1998.

Hansen, D. R. and Mauersberger, K.: Laboratory studies of the nitric acid trihydrate: Implications for the south polar stratosphere, Geophys. Res. Lett., 15, 855-858, 1988.

Hostetler, C. A., Liu, Z., Reagan, J., Vaughan, M., Winker, D., Osborn, M., Hunt, W. H., Powell, K. A., and Trepte, C.: CALIOP Algorithm Theoretical Basis Document- Part 1: Calibration and Level 1 Data Products, PC-SCI-201, NASA Langley Research Center, Hampton, VA, USA, online available at: http://www-calipso.larc.nasa.gov/resources/ project_documentation.php, 2006.

Höpfner, M., Pitts, M. C., and Poole, L. R.: Comparison between CALIPSO and MIPAS observations of polar stratospheric clouds, J. Geophys. Res., doi:10.1029/2009JD012114, in press, 2009. 
Hu, R.-M., Carslaw, K. S., Hostetler, C., Poole, L. R., Luo, B., Peter, T., Füeglistaler, S., McGee, T. J., Burris, J. F.: Microphysical properties of wave polar stratospheric clouds retrieved from lidar measurements during SOLVE/THESEO 2000, J. Geophys. Res., 107 (D20), 8294, doi:10.1029/2001JD001125, 2002.

Hunt, W. H., D. M. Winker, M. A. Vaughan, K. A. Powell, et al:: CALIPSO lidar description and performance assessment, J. Atmos. Oceanic Technol., 26, 1214-1228, doi:10.1175/2009JTECHA1223.1, 2009.

Larsen, N., Knudsen, B. M., Svendsen, S. H., Deshler, T., Rosen, J. M., Kivi, R., Weisser, C., Schreiner, J., Mauerberger, K., Cairo, F., Ovarlez, J., Oelhaf, H., and Spang, R.: Formation of solid particles in synoptic-scale Arctic PSCs in early winter 2002/2003, Atmos. Chem. Phys., 4, 2001-2013, 2004.

List, R. J.: Smithsonian Meteorological Tables, 6th edition, Smithsonian Institution Press, Washington, DC, USA, 527 pp., 1984.

Liu, L. and Mishchenko, M. I.: Constraints on PSC particle microphysics derived from lidar observations, J. Quant. Spectrom. Rad. T., 70, 817-831, 2001.

Liu, Z., Vaughan, M. A., Winker, D. M.., Hostetler, C. A., Poole, L. R., Hlavka, D., Hart, W., and McGill, M.: Use of probability distribution functions for discriminating between cloud and aerosol in lidar backscatter data, J. Geophys. Res., 109, D15202, doi:10.1029/2004JD004732, 2004.

Lowe, D. and A. R. MacKenzie, Polar stratospheric cloud microphysics and chemistry, J. Atmos. Solar-Terr. Phys., 70, 13-40, 2008.

Manney, G. L., Daffer, W. H., Zawodny, J. M., et al.: Solar occultation satellite data and derived meteorological products: Sampling issues and comparisons with Aura Microwave Limb Sounder, J. Geophys. Res., 112, D24S50, doi:10.1029/2007JD008709, 2007.

Massoli, P., Maturilli, M., and Neuber, R.: Climatology of Arctic polar stratospheric clouds as measured by lidar in Ny- Ålesund, Spitsbergen $\left(79^{\circ} \mathrm{N}, 12^{\circ} \mathrm{E}\right)$, J. Geophys. Res., 111, D09206, doi:10.1029/2005JD005840, 2006.

McGill, M. J., Vaughan, M. A., Trepte, C. R., Hart, W. D., Winker, D. M., and Kuehn, R.: Airborne validation of spatial properties measured by the CALIPSO lidar, J. Geophys. Res., 112, D20201, doi:10.1029/2007JD008768, 2007.

Mishchenko, M. I., and Travis, L. D.: Capabilities and limitations of a current Fortran implementation of the T-matrix code for randomly oriented, rotationally symmetric scatterers, J. Quant. Spectr. Rad. T., 60, 309-324, 1998.
Northway, M. J., Gao, R. S., Popp, P. J., Holecek, J. C., Fahey, D. W., et al.: An analysis of large $\mathrm{HNO}_{3}$-containing particles sampled in the Arctic stratosphere during the winter of 1999/2000, J. Geophys. Res., 107(D20), 8298, doi:10.1029/2001JD001079, 2002.

Pitts, M. C., Thomason, L. W., Poole, L. R., and Winker, D. M.: Characterization of polar stratospheric clouds with spaceborne lidar: CALIPSO and the 2006 Antarctic season, Atmos. Chem. Phys., 7, 5207-5228, 2007, http://www.atmos-chem-phys.net/7/5207/2007/.

Poole, L. R. and McCormick, M. P.: Airborne lidar observations of Arctic polar stratospheric clouds: Indications of two distinct growth stages, Geophys. Res. Lett., 15, 21-23, 1988.

Poole, L. R. and Pitts, M. C.: Polar stratospheric cloud climatology based on Stratospheric Aerosol Measurement II observations from 1978 to 1989, J. Geophys. Res., 99, 13083-13089, 1994.

Powell, K. A., C. A. Hostetler, Z. Liu, M. A. Vaughan, et al.: CALIPSO lidar calibration algorithms: Part I- Nighttime 532-nm parallel and 532-nm perpendicular channel, J. Atmos. Oceanic Technol., 26, doi:10.1175/2009JTECHA1242.1, in press, 2009.

Shibata, T., Shiraishi, K., Adachi, H., Iwasaka, Y., and Fujiwara, M.: On the lidar-observed sandwich structure of polar stratospheric clouds (PSCs) 1. Implications for the mixing state of the PSC particles, J. Geophys. Res., 104, 21603-21611, 1999.

Stephens, G. L., Vane, D. G., Boain, R. J., Mace, G. G., et al.: The CloudSat mission and the A-Train: A new dimension of spacebased observations of clouds and precipitation, B. Am. Meteor Soc., 83, 1771-1790, 2002.

Toon, O. B., Browell, E. V., Kinne, S., and Jordan, J.: An analysis of lidar observations of polar stratospheric clouds, Geophys. Res. Lett., 17, 393-396, 1990.

Toon, O., Tabazadeh, A., Browell, E., and Jordan, J.: Analysis of lidar observations of Arctic polar stratospheric clouds during January 1989, J. Geophys. Res., 105(D16), 20589-20615, 2000.

Weisser, C., Mauersberger, K., Schreiner, J., Larsen, N., Cairo, F., Adriani, A., Ovarlez, J., and Deshler, T.: Composition analysis of liquid particles in the Arctic stratosphere under synoptic conditions, Atmos. Chem. Phys., 6, 689-696, 2006, http://www.atmos-chem-phys.net/6/689/2006/.

Winker, D. M., McGill, M., and Hunt, W. H.: Initial performance assessment of CALIOP, Geophys. Res. Lett., 34, L19803, doi:10.1029/2007GL030135,2007. 